\title{
On the recirculating flow of three-dimensional asymmetric bluff bodies
}

\author{
Antoine Legeai ${ }^{1} \cdot$ Olivier Cadot $^{1}[$
}

Received: 24 June 2020 / Revised: 13 October 2020 / Accepted: 19 October 2020 / Published online: 10 November 2020

(c) The Author(s) 2020

\begin{abstract}
The paper investigates the role of geometrical asymmetric modifications of a rectangular flat-backed body on the properties of the recirculating flow at a Reynolds number $R e=1.8 \times 10^{5}$. The reference model has two reflectional symmetries denoted $s_{y}$ and $s_{z}$ in both spanwise directions. The flow is subjected to the static instability that leads to two mirrored wake states breaking the symmetry $s_{y}$. Two families of geometrical variation of the fore-body and after-body are studied, each breaking one of the reflectional symmetries of the reference model. Geometrical modifications that preserve $s_{y}$ evidence possibilities of bistable dynamics suppression although the static instability persists. Geometrical modifications that do not preserve $s_{y}$ produces a large unbalance of both wake states in accordance to recent observations on real cars (Bonnavion et al. in J Wind Eng Ind Aerodyn 184:77-89, 2019). Results offer perspectives for potential drag reduction induced by appropriate coupling of bluff body geometry and wake state selection.
\end{abstract}

\section{Graphic abstract}

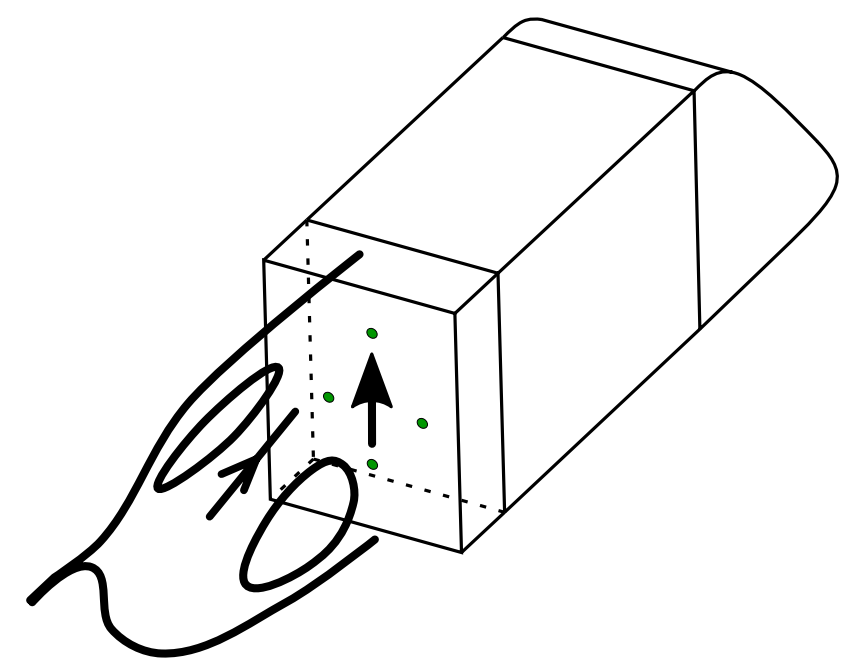

\section{Introduction}

Flat-backed three-dimensional bluff bodies are known to develop a static instability responsible for a permanent symmetry breaking or deviation of the wake. For Reynolds

Olivier Cadot

cadot@liverpool.ac.uk

1 University of Liverpool, Liverpool, UK numbers beyond the laminar regime where turbulence restores symmetries, very long time dynamics are observed. They are associated with random rotations of the deviated wake for circular base bodies (see Rigas et al. 2014, 2015 for seminal papers) and random switching of two opposite wake deviations (bistability) for rectangular base bodies (see Grandemange et al. 2012, 2013b for seminal papers). In the later case, the wake deviations are observed in the major direction of the rectangular base (Grandemange et al. 2013a; 
Bonnavion and Cadot 2018). Two cases need to be distinguished whether the deviations are in a perpendicular or parallel direction to the ground, respectively, referring to as the $z$ and $y$-instability following (Grandemange et al. 2013a).

The $z$-instability has first been reported by Grandemange et al. (2013a). For sufficiently high ground clearance (larger than only $7 \%$ of the body width), it produces two possible wake states globally deviated towards the top or the ground, with a positive ( $P$ state) or negative ( $N$ state) vertical base pressure gradient, respectively. This instability has been studied in several academic experiments in a Reynolds number range based on the body height $H$, of $R e \approx 4.5 \times 10^{4}$ $-6.7 \times 10^{5}$ in Grandemange et al. (2013a), Schmidt et al. (2018) and Bonnavion and Cadot (2018), and numerically at $R e=5.2 \times 10^{4}$ in Dalla Longa et al. (2019). Although there is no symmetry in the vertical direction (due to the ground and the body supports), both states present opposite vertical base pressure coefficient gradients $\left\langle\frac{\partial c_{p}}{\partial z}\right\rangle_{P}=-\left\langle\frac{\partial c_{p}}{\partial z}\right\rangle_{N}$ with a magnitude that depends on the specific geometry but always in the range $0.1 H^{-1}$ to $0.2 H^{-1}$. The consequence on the body aerodynamic loading is substantial and the lift coefficient difference between both $N$ and $P$ states has been measured to be $\left\langle c_{L}\right\rangle_{N}-\left\langle c_{L}\right\rangle_{P}=0.04$ in Bonnavion and Cadot (2018) for the Ahmed body. The same value is also reported for the side force coefficient for the Windsor body in Perry et al. (2016) as well as in Bonnavion and Cadot (2018) when the body base is wider than tall thus leading to the $y$-instability (i.e. exhibiting permanent non-zero horizontal base pressure gradient). These results suggest that the ground has no influence on the aerodynamic loading induced by the wake $z$-instability.

The $z$-instability has been recently shown to be a common feature of real minivans at $R e \approx 5 \times 10^{6}$ with rotating wheels and road effect (Bonnavion et al. 2019). On the contrary to the academic geometries cited above, both $N$ and $P$ vertical gradients are not opposite but with $\left\langle\frac{\partial c_{p}}{\partial z}\right\rangle_{P} \approx-2\left\langle\frac{\partial c_{p}}{\partial z}\right\rangle_{N}$. The magnitude of the gradient depends on the vehicle type but still takes values within the range $0.1 H^{-1}$ to $0.2 H^{-1}$. The effect on the lift is similar to that observed for the academic geometry with $\left\langle c_{L}\right\rangle_{N}-\left\langle c_{L}\right\rangle_{P}=0.036$. The potential of direct passive control of the z-instability is demonstrated by selecting the state $P$ that is associated with less drag than the state $N$. Their interpretation is based on the induced drag contribution: the lower drag configuration corresponds to the wake state that reduces the cross-flow force produced by the body shape as reported in Bonnavion and Cadot (2018). This reduction is due to the vertical base pressure gradient imposed by the static instability that beneficially modifies the rear top and bottom pressure distribution of the body.

Therefore, the wake state selection of a ground vehicle subjected to the $z$-instability appears to be a lever for drag reduction. since the vertical wake orientation in the $z$-direction of a vehicle is known to affect the drag, and that classical aerodynamics optimisation (Grandemange et al. 2015) is performed by finding the best couple of angles for the spoiler and the diffuser, respectively located at the top and bottom rear edges of the body. So far, there have not been much parametric studies to show how does the body geometry itself (fore- or after-body shapes) selects the state of a wake subjected to the $z$-instability. Most fundamental studies investigate simplified ground vehicles subjected to a (horizontal) $y$-instability and as they concentrate on rear geometrical modifications that respect the reflectional body symmetry (Littlewood and Passmore 2010; Grandemange et al. 2015; Pavia et al. 2016; Barros et al. 2017; Bonnavion and Cadot 2019), the wake instability direction is always perpendicular to the direction of the geometrical modifications. From the real minivans study of Bonnavion et al. (2019), it seems relevant for the industrial applications to investigate fundamentally the coupling between the static $z$ instability and geometrical modifications that respect the $y \rightarrow-y$ reflectional symmetry (i.e. when the instability and the geometrical variations are in the same direction). There are only few studies corresponding to this case, although their wake are always subjected to the $y$-instability. Grandemange et al. (2014) was the first to show that a small vertical cylinder in the recirculating flow at the rear of the Ahmed body subjected to the $y$-instability affects base suction and wake states selection. Brackston et al. (2016) have shown the efficient authority to select wake states using vertical flaps at both shortest rear edges of the Ahmed body subjected to the $y$-instability. Similarly Varney et al. (2018) produced vertical asymmetric tapers at both shortest rear edge of the Windsor body subjected to the $y$-instability (Perry et al. 2016), however, the paper does not mention the presence of the static mode associated with the $y$-instability. For these rare studies performing geometrical modifications in the same direction of the instability, the fore-body geometry has never been addressed.

The paper first addresses the question: why the $z$-instability for industrial geometries does not produce antisymmetric vertical gradients as for simple parallelepiped bodies? Since the observation of perfect anti-symmetric pressure gradients with parallelepiped bodies excludes ground effect, it is necessary to investigate the body geometry itself as a possible cause for the discrepancy. Second, how does the body geometry, especially the fore-body influence the wake states selection? For these purposes, the present experiment investigates the base pressure gradient of a parallelepiped body of rectangular cross section subjected to some geometrical variations in both lateral directions from a symmetric to an asymmetric fore-body or after-body and with no ground proximity. 
(a)

(b)

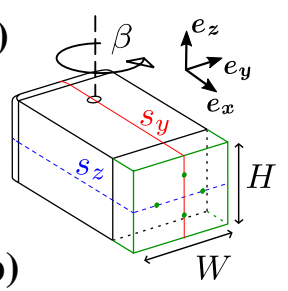

(d)

(c)
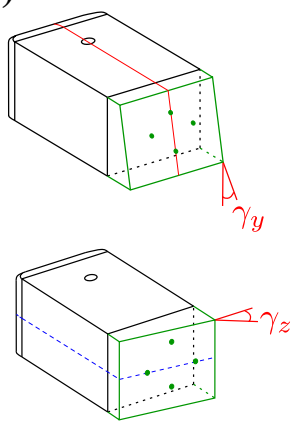

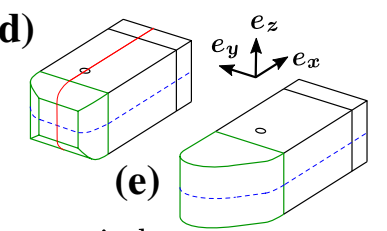

wind

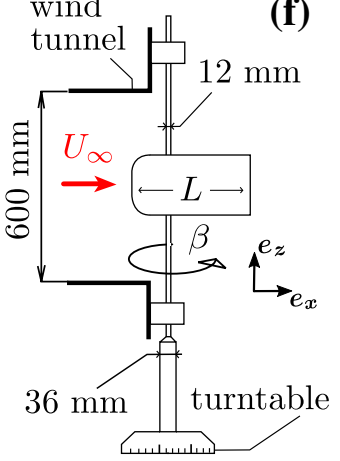

Fig. 1 Model geometries and experimental setup. a, $\mathbf{d}$ Reference case with the two reflectional symmetries $s_{y}$ and $s_{z}$. b, c Base slant modifications of the reference case. $\mathbf{d}$ Fore-body modification of the reference case. $\mathbf{f}$ Reference case in the wind tunnel

\section{Experimental geometries}

The bodies in Fig. 1 are geometrical variations of the square-back Ahmed body drawn in Fig. 1a, d, referred to as the reference case for the remainder of the paper. The reference case has for characteristic dimensions $(H) \times\left(W=\frac{4}{3} H\right) \times(L=2.5 H)$ with $H=180 \mathrm{~mm}$. Two families of geometries are investigated, each breaking one of the reflectional symmetries of the reference geometry with respect to the symmetry planes $s_{y}$ and $s_{z}$ shown in Fig. 1a, d. The geometries that preserve $s_{y}$ (Fig. 1b) are made of the reference fore-body and a slanted base with a variable angle $\gamma_{y}$. The geometries that preserve $s_{z}$ are made of either the reference fore-body and a slanted base with a variable angle $\gamma_{z}$ (Fig. 1c) or an asymmetric fore-body and a square-back (Fig. 1e). To realise all the geometries, parts highlighted with green color in Fig. 1 are interchangeable. There are 10 parts in total for the present work.

As shown in Fig. 1f, the body is supported by a vertical cylinder of $12 \mathrm{~mm}$ in diameter and placed in an open jet blow-down wind tunnel with dimensions $600 \times 1200 \mathrm{~mm}$. The wind speed is set to $U_{\infty}=20 \mathrm{~m} \mathrm{~s}^{-1}$ with a free stream turbulent intensity of $1 \%$ giving a flow Reynolds number $\mathrm{Re}=1.8 \times 10^{5}$ based on the body height $H$. The body can rotate accurately to set the yaw angle $\beta$ thanks to a manual turntable. Geometrical modifications by changing any front or rear parts imply a loss on the accuracy of the datum of the yaw angle measurement of about $\delta \beta= \pm 0.25^{\circ}$.

The minimal set of four pressure measurements at the rear of the body (displayed as green dots in Fig. 1) is used to compute the pressure gradients in the horizontal and vertical

directions with a simple difference. Pressure taps are symmetrically distributed around the surface base centre and separated by $H / 2$ vertically and $W / 2$ horizontally. The acquisition is sampled at $1 \mathrm{kHz}$ for each tap using a pressure scanner ZOC 33 from Scanivalve, demultiplexed with Labview and a NI acquisition board. Pressure time series $p(t)$ are lowpass filtered using a sliding window of 200 points corresponding to a cutoff frequency $f_{c}=0.45 U_{\infty} / H$. They are translated into pressure coefficient $c_{p}=2\left(p-p_{\infty}\right) /\left(\rho U_{\infty}^{2}\right)$, where $\rho$ and $p_{\infty}$ are respectively the air density and the static pressure at the wind tunnel aperture. In the following, we use the notations $g_{y}=H \frac{\partial c_{p}}{\partial y}$ and $g_{z}=H \frac{\partial c_{p}}{\partial z}$ for the two dimensionless components of the base pressure coefficient gradients computed with this minimal set. As there is clear consensus about the relationship between the base pressure gradients orientation and the recirculating flow topology (Barros et al. 2017; Bonnavion and Cadot 2018; Bonnavion et al. 2019; Dalla Longa et al. 2019; Grandemange et al. 2013a; Perry et al. 2016; Schmidt et al. 2018), wake states are accordingly illustrated as in Fig. 2 and elsewhere in the paper to clarify the separated flow asymmetry as deduced from the pressure gradients. Finally, the base suction $C_{B}$ is obtained from minus the four pressure taps averaging.

\section{Results and discussion}

Gradient dynamics and statistics are shown for the reference case in Fig. 2. As found in Grandemange et al. (2013a), the horizontal component of the gradient switches randomly
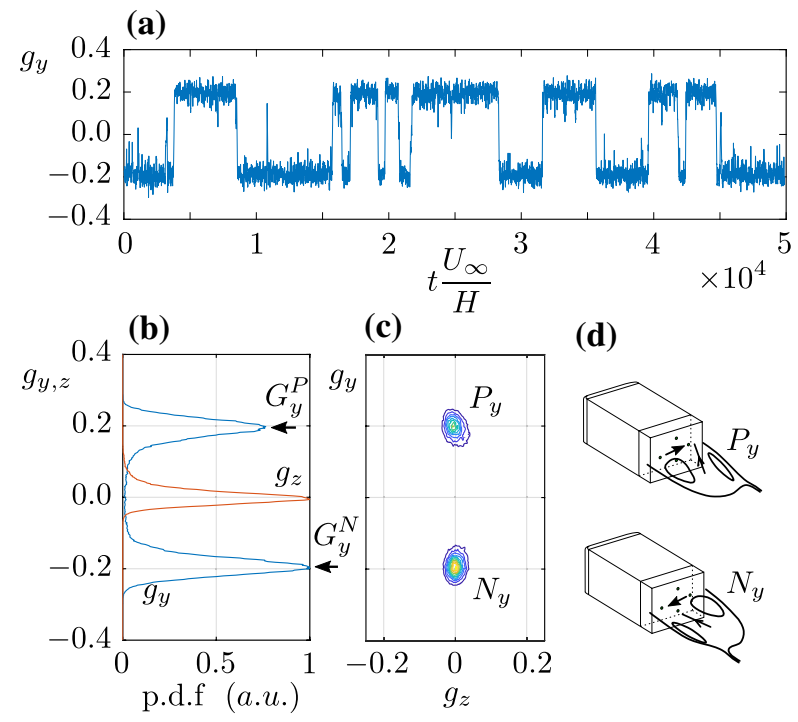

Fig. 2 Gradient dynamics (a) and statistics (b, c) of the reference case. Illustrations (d) of the mean wake corresponding to each gradient state $P_{y}$ or $N_{y}$ 
(Fig. 2a) between two symmetry breaking states denoted $P_{y}$ and $N_{y}$ identified as most probable events in each statistics of the components (Fig. 2b) and joint statistics (Fig. 2c). According to Grandemange et al. (2013a), the two corresponding mean wake states are illustrated in Fig. 2c with opposite $G_{y}^{P}$ and $G_{y}^{N}$ given by the two most probable values (Fig. 2b). The instability is associated with an asymmetry in the $y$-direction. Note that in the absence of a ground proximity, definitions of $y$ - and $z$-instabilities referring to as asymmetries, respectively, parallel and perpendicular to the ground as used in Grandemange et al. (2013a) are not appropriate for the present study. Instead, it is preferable to refer to as the major axis of the rectangular base as introduced in Bonnavion and Cadot (2018) for the direction of the instability. For all body geometries presented below, the bistable regime is sought by varying the yaw angle by steps of $\Delta \beta=0.05^{\circ}$ with acquisition of 300-s duration for each yaw. The bistable regime is found when the two states are observable during the dynamics. It allows to compare unambiguously the aerodynamic performance of the two states as they are obtained in exactly the same flow configuration. For all the different body geometries, the bistable regime is always found within a small range that never exceeds $0.4^{\circ}$ in yaws.

The first geometrical modifications considered in Fig. 3 preserve the reflectional symmetry $s_{y}$. For this set of experiments the body is aligned with the incoming flow. As can be seen in Fig. 3a, the bistable dynamics of the horizontal pressure gradient remains observable for base slant inclinations $\gamma_{y}<15^{\circ}$. In this slant angles range, the gradient modulus is almost constant with $G \approx 0.2$ and a small negative vertical gradient $G_{z}$ is produced by the inclined base. At $\gamma_{y}=16^{\circ}$,

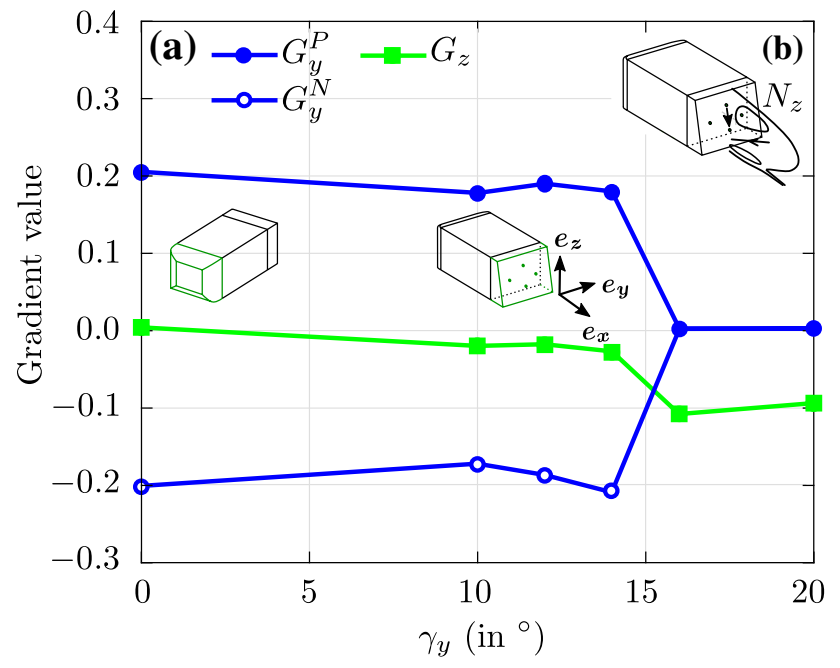

Fig. 3 Most probable values $G_{y}^{P}, G_{y}^{N}, G_{z}$ of the pressure gradient (a) as a function of the base slant angle $\gamma_{y}$. Illustration of the wake state (b) for $\gamma_{y}>15^{\circ}$ a clear transition occurs, where the bistable dynamics is replaced by a single wake state with a substantial vertical gradient $G_{z} \approx-0.1$. The sudden increase of the vertical component at $\gamma_{y}=16^{\circ}$ is likely to be due to a change of orientation of the direction of the instability. The inset Fig. $3 \mathrm{~b}$ illustrates the wake associated with the observed gradient. The flow is similar to that of Barros et al. (2017) for their reference square-back body. We then followed their methodology to retrieve the bistability from the $\gamma_{y}=16^{\circ}$ slant configuration by obstructing the flow with square cylinders of diameter $d / H=0.033$ at the base edge pointed by the gradient. For the passive disturbances configuration shown in Fig. 4, a multistable dynamics is retrieved as shown in Fig. $4 \mathrm{a}-\mathrm{c}$, involving $P_{y}$ and $N_{y}$ states such as for the reference case but with larger intensity $\left(G_{y}^{p}=-G_{y}^{N} \approx 0.3, G_{z}=0\right)$ and the previous $N_{z}$ state $\left(G_{z} \approx-0.1, G_{y}=0\right)$. The 3 wake states are illustrated in Fig. 4d. The tristable dynamics observed in Fig. 4 must be induced by the slant angle since in Barros et al. (2017) only bistable dynamics are triggered with this passive control method on the straight square-back geometry.

The second series of geometrical modifications break the same symmetry as the static instability because they do not preserve the symmetry $s_{y}$. This is the situation met for real minivans where the body shape has no symmetry in the vertical direction that is also the direction of the instability (Bonnavion et al. 2019). When aligned with the wind ( $\beta=0)$, both geometries select permanently the wake state with a negative horizontal pressure gradient, $G_{y}^{N}=-0.2$ as depicted in Fig. 5a and $G_{y}^{N}=-0.24$ in Fig. 5 b. There is

(a)
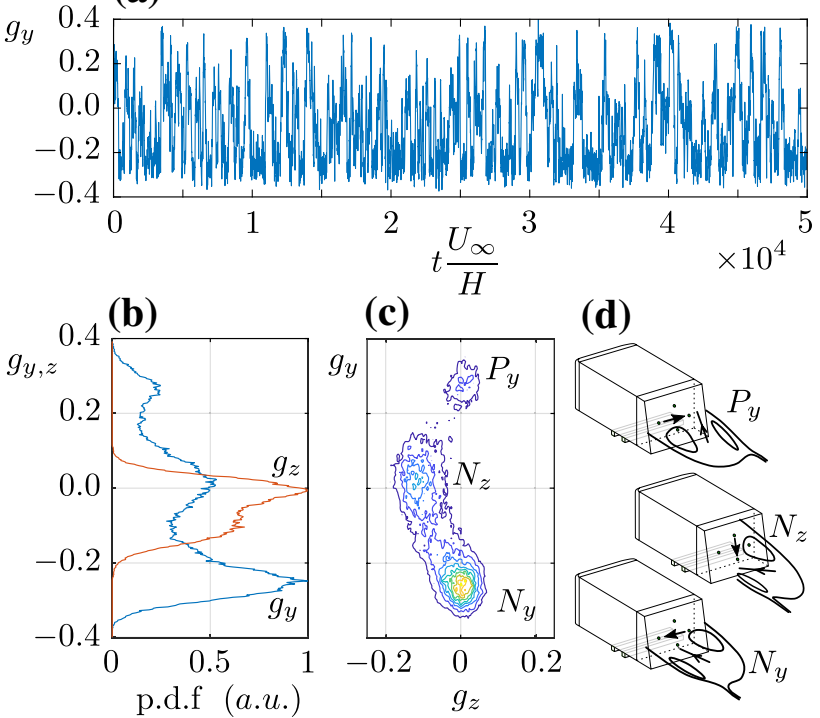

(d)

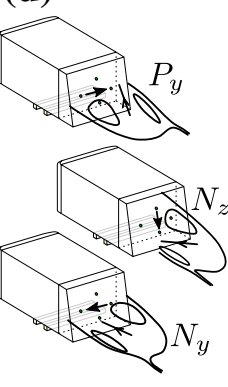

Fig. 4 Gradient dynamics (a) and statistics (b, c) of the slanted base body $\gamma_{y}=16^{\circ}$ with additional passive devices. Illustrations (d) of the mean wake corresponding to each gradient state $P_{y}, N_{y}$ or $N_{z}$ 


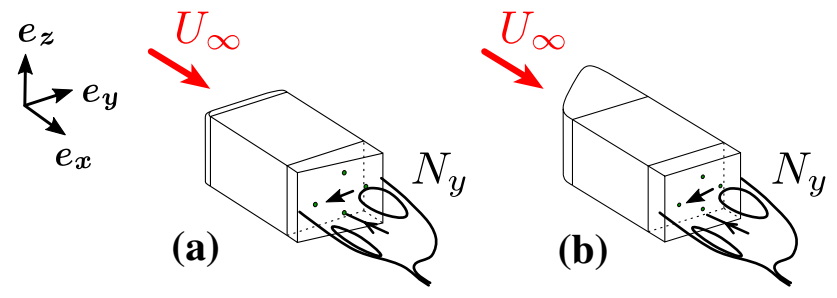

Fig. 5 Wake state illustrations in aligned configuration for (a) slanted base (any $\gamma_{z} \neq 0$ ) and (b) asymmetric fore-body

no trivial relationship between the base gradient orientation and the body shape. We can nevertheless see that the base aspect ratio plays a role for the slanted base cases since the gradient points to the longer side in Fig. $3 \mathrm{~b}$ while it points to the shorter side in Fig. 5a. It is likely that in the first case the slant angle produces a $\pi / 2$ rotation of the wake similarly to the roof/underbody steady disturbances of Barros et al. (2017) or the body pitch variation of Bonnavion and Cadot (2018) for an Ahmed body subjected to the $y$-instability. This effect is actually generally observed when a squareback base subjected to $y$-instability (either the Windsor or the Ahmed body) is modified with top and bottom rear, tapers (Perry et al. 2016) or boat tails (Bonnavion and Cadot 2019). For instance, the presence of the bottom diffuser of the Windsor body studied in Luckhurst et al. (2019) has definitely selected a $P_{z}$ state compared to the square-back Windsor model (Perry et al. 2016). Bonnavion and Cadot (2018) actually identify 2 mechanisms of wake orientation with body inclination, a wake adaptation when the modification respect the symmetry in the instability direction (as in Fig. 3b) and a wake state selection when it breaks the symmetry in the instability direction (as in Fig. 5a). For each case of $\beta=0$ in Fig. 5, a positive yaw is necessary to recover a bistable dynamics whose corresponding values $\beta$ are reported in table 1 .

The most probable gradient observed during the bistable dynamics are plotted in Fig. 6. For the asymmetric fore-body geometry, the two gradients are not opposite to each other as shown by the diamond symbols. This situation seems similar to that of real minivan and indicates that the forebody shape of the real minivan car is responsible for the disequilibrium of the two wake states pressure gradients. A

Table 1 Absolute yaw range for which bistability is observed. The angle $\beta$ is defined from the symmetry axis of the wind tunnel

\begin{tabular}{llll}
\hline Geometry & $\beta$ & $\left\langle C_{B}\right\rangle_{N}$ & $\left\langle C_{B}\right\rangle_{P}$ \\
\hline Figure 1a (reference) & $0^{\circ} \pm 0.15^{\circ}$ & 0.153 & 0.153 \\
Figure 1c $\left(\gamma_{z}=10^{\circ}\right)$ & $3^{\circ} \pm 0.1^{\circ}$ & 0.142 & 0.134 \\
Figure 1c $\left(\gamma_{z}=20^{\circ}\right)$ & $6.1^{\circ} \pm 0.2^{\circ}$ & 0.154 & 0.144 \\
Figure 1e $\left(\gamma_{z}=0^{\circ}\right)$ & $2^{\circ} \pm 0.025^{\circ}$ & 0.141 & 0.148 \\
\hline
\end{tabular}

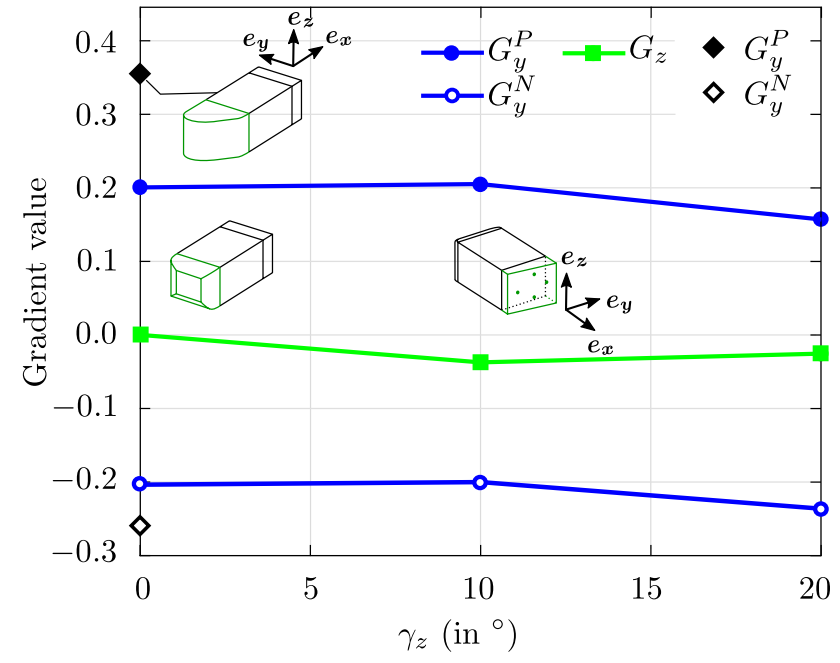

Fig. 6 Most probable values $G_{y}^{P}, G_{y}^{N}, G_{z}$ of the pressure gradient as a function of the base slant angle $\gamma_{z}$ for the asymmetric and symmetric fore-bodies as depicted in the plot

global negative shift of the gradients of both states can be produced with a $\gamma_{z}$ slant after-body of $20^{\circ}$ as shown in Fig. 6 with the symmetric fore-body. We can see from Table 1 that the asymmetric modifications of either the front or the rear are beneficial for the base suction. Interestingly, the reduction depends on the selected wake states, and the better base suction reduction is observed for the $P$ states for slanted base geometries (Fig. 1c) and the $N$ state for the asymmetric fore-body (Fig. 1e). A plausible explanation of this effect is provided by Bonnavion et al. (2019) arguing that the wake orientation that reduces the lift produced by the body shape corresponds to the lower drag. The case with the asymmetric fore-body seems to exactly corresponds to this. As the forebody asymmetry is likely to produce a negative cross-flow force in the $y$-direction in Fig. 5b, a negative base pressure gradient $N_{y}$ should affect the pressure around the base by reducing the total cross-flow force of the body as reported in Bonnavion and Cadot (2018). For consistency, the crossflow force produced by the shape in Fig. 5a has to be positive in the $y$-direction for the positive base pressure gradient to reduce the total cross-flow force.

As the fore-body loading remains almost unchanged during the bistable wake dynamics, the base suction variation directly impacts the total drag of the body $\Delta C_{b}=\Delta C_{d}$. This variation is observed to be of order $\Delta C_{b} \approx 0.01$.

\section{Conclusion}

This academic experiment made in the absence of a ground proximity shows how the asymmetries of the front and the rear of the body modify the intensity of the base pressure 
gradient of the two wake states. Geometrical modification that do not preserve the reflectional symmetry in the instability direction (that is observed for real minivans) produces a large unbalance in the wake states gradient when located at the fore-body. When located at the rear, the geometrical modification produces a global shift of both gradients. The paper points out the possible consequence for the induced drag of the gradient orientation that can either increase or decrease the cross flow force (i.e. the lift force for real minivans) depending on the wake state selected by the geometry. For future development, it would then be relevant to produce bluff body shapes that could take favour of the instability to reduce drag with appropriate base pressure gradient orientation and intensity.

Acknowledgements The authors are grateful to Guillaume Bonnavion for useful discussions and his critical reading of the paper as well as to the mechanical workshop staff of the University for designing the models.

Open Access This article is licensed under a Creative Commons Attribution 4.0 International License, which permits use, sharing, adaptation, distribution and reproduction in any medium or format, as long as you give appropriate credit to the original author(s) and the source, provide a link to the Creative Commons licence, and indicate if changes were made. The images or other third party material in this article are included in the article's Creative Commons licence, unless indicated otherwise in a credit line to the material. If material is not included in the article's Creative Commons licence and your intended use is not permitted by statutory regulation or exceeds the permitted use, you will need to obtain permission directly from the copyright holder. To view a copy of this licence, visit http://creativecommons.org/licenses/by/4.0/.

\section{References}

Barros D, Borée J, Cadot O, Spohn A, Noack BR (2017) Forcing symmetry exchanges and flow reversals in turbulent wakes. J Fluid Mech 829:R1

Bonnavion G, Cadot O (2018) Unstable wake dynamics of rectangular flat-backed bluff bodies with inclination and ground proximity. J Fluid Mech 854:196-232

Bonnavion G, Cadot O (2019) Boat-tail effects on the global wake dynamics of a flat-backed body with rectangular section. J Fluids Struct 89:61-71

Bonnavion G, Cadot O, Herbert V, Parpais S, Vigneron R, Délery J (2019) Asymmetry and global instability of real minivans' wake. J Wind Eng Ind Aerodyn 184:77-89
Brackston RD, García De La Cruz J, Wynn A, Rigas G, Morrison JF (2016) Stochastic modelling and feedback control of bistability in a turbulent bluff body wake. J Fluid Mech 802:726-749. https ://doi.org/10.1017/jfm.2016.495

Dalla Longa L, Evstafyeva O, Morgans A (2019) Simulations of the bi-modal wake past three-dimensional blunt bluff bodies. J Fluid Mech 866:791-809

Grandemange M, Gohlke M, Cadot O (2012) Reflectional symmetry breaking of the separated flow over three-dimensional bluff bodies. Phys Rev E 86:035302

Grandemange M, Gohlke M, Cadot O (2013a) Bi-stability in the turbulent wake past parallelepiped bodies with various aspect ratios and wall effects. Phys Fluids 25:95-103

Grandemange M, Gohlke M, Cadot O (2013b) Turbulent wake past a three-dimensional blunt body. Part 1. Global modes and bistability. J Fluid Mech 722:51-84

Grandemange M, Gohlke M, Cadot O (2014) Turbulent wake past a three-dimensional blunt body. Part 2. Experimental sensitivity analysis. J Fluid Mech 752:439-461

Grandemange M, Cadot O, Courbois A, Herbert V, Ricot D, Ruiz T, Vigneron R (2015) A study of wake effects on the drag of the Ahmed squareback model at the industrial scale. J Wind Eng Ind Aerodyn 145:282-291

Littlewood R, Passmore M (2010) The optimization of roof trailing edge geometry of a simple squareback. SAE technical paper series 2010-01-0510

Luckhurst S, Varney M, Xia H, Passmore M, Gaylard A (2019) Computational investigation into the sensitivity of a simplified vehicle wake to small base geometry changes. J Wind Eng Ind Aerodyn 185:1-15. https://doi.org/10.1016/j.jweia.2018.12.010

Pavia G, Passmore M, Gaylard A (2016) Influence of short rear end tapers on the unsteady base pressure of a simplified ground vehicle. SAE Tech Pap Ser. https://doi.org/10.4271/2016-01-1590

Perry A, Pavia G, Passmore M (2016) Influence of short rear end tapers on the wake of a simplified square-back vehicle: wake topology and rear drag. Exp Fluids 57(11):169

Rigas G, Oxlade A, Morgans A, Morrison J (2014) Low-dimensional dynamics of a turbulent axisymmetric wake. J Fluid Mech 755:159

Rigas G, Morgans A, Brackston RD, Morrison J (2015) Diffusive dynamics and stochastic models of turbulent axisymmetric wakes. J Fluid Mech 778:R2

Schmidt HJ, Woszidlo R, Nayeri CN, Paschereit CO (2018) The effect of flow control on the wake dynamics of a rectangular bluff body in ground proximity. Exp Fluids 59(6):107

Varney M, Passmore M, Gaylard A (2018) Parametric study of asymmetric side tapering in constant cross wind conditions. SAE Int J Passeng Cars Mech Syst 11:3

Publisher's Note Springer Nature remains neutral with regard to jurisdictional claims in published maps and institutional affiliations. 